Rapid Reviews COVID-19

\title{
Reviews of "The effect of influenza vaccination on trained immunity: impact on COVID-19"
}

Ming-Jim Yang ${ }^{1}$, Samer Singh ${ }^{2}$

${ }^{1}$ University of Florida, Hospital Medicine, USA,

${ }^{2}$ Banaras Hindu University Institute of Medical Sciences, Centre of Experimental Medicine and Surgery, India

Published on: Nov 25, 2020

DOI: $10.1162 / 2 \mathrm{e} 3983 f 5.95730 \mathrm{ea} 2$

License: Creative Commons Attribution 4.0 International License (CC-BY 4.0). 
To read the original manuscript, click the link above.

Summary of Reviews: This study explores whether the influenza vaccine elicits protection against SARS$\mathrm{CoV}-2$ via trained immunity. While some of the study's findings are compelling, the work's major claims are generally unsubstantiated by the data offered.

\title{
Reviewer 1 (Ming-Jim Yang) |
}

\section{Reviewer 2 (Samer Singh) |}

\author{
RR:C19 Strength of Evidence Scale Key. \\ $\square \square \square \square \square=$ Misleading \\ $\square \square \square \square$ = Not Informative \\ $\square \square \square \square$ = Potentially Informative \\ पढ口 $\square=$ Reliable

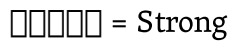

To read the reviews, click the links below. 